OPEN ACCESS

Edited by: Giuseppe Esposito, Sapienza University of Rome, Italy

Reviewed by: Yang Liu, Peking University Third Hospital, China Yun K. Tam,

Sinoveda Canada Inc., Canada

*Correspondence: Bücker R. roland-felix.buecker@charite.de

Specialty section: This article was submitted to Ethnopharmacology,

a section of the journal

Frontiers in Pharmacology

Received: 11 December 2020 Accepted: 19 February 2021 Published: 15 April 2021

Citation:

Lobo de Sá FD, Heimesaat MM, Bereswill S, Nattramilarasu PK, Schulzke J D and Bücker R (2021) Resveratrol Prevents Campylobacter jejuni-Induced Leaky gut by Restoring

Occludin and Claudin-5 in the

Paracellular Leak Pathway.

Front. Pharmacol. 12:640572.

doi: 10.3389/fphar.2021.640572

\section{Resveratrol Prevents Campylobacter jejuni-Induced Leaky gut by Restoring Occludin and Claudin-5 in the Paracellular Leak Pathway}

\author{
F. D. Lobo de Sá ${ }^{1}$, M. M. Heimesaat ${ }^{2}$, S. Bereswill ${ }^{2}$, P. K. Nattramilarasu ${ }^{1}$, J. D. Schulzke ${ }^{1}$ and \\ R. Bücker ${ }^{1 *}$
}

${ }^{1}$ Medical Department, Division of Gastroenterology, Infectious Diseases, Rheumatology, Nutritional Medicine/Clinical Physiology, Charité-Universitätsmedizin Berlin, Berlin, Germany, ${ }^{2}$ Institute of Microbiology, Infectious Diseases, and Immunology, Gastrointestinal Microbiology Research Group, Charité - Universitätsmedizin Berlin, Berlin, Germany

Campylobacter jejuni is a bacterial human pathogen causing gastroenteritis and sequelae like irritable bowel syndrome. Epidemiologists count the human campylobacteriosis by $C$. jejuni as the most common foodborne zoonosis and bacterial diarrheal disease worldwide. Based on bioinformatics predictions for potential protective compounds in campylobacteriosis, the question was raised whether the plant-based polyphenol resveratrol is sufficient to attenuate intestinal epithelial damage induced by $C$. jejuni. We investigated this by performing experimental infection studies in an epithelial cell culture and the secondary abiotic IL-10 ${ }^{-/-}$mouse model. In C. jejuni-infected human colonic HT29/B6 cell monolayers, transepithelial electrical resistance (TER) was decreased and the paracellular marker flux of fluorescein (332 Da) increased. Concomitantly, the tight junction (TJ) proteins occludin and claudin- 5 were re-distributed off the tight junction domain. This was accompanied by an increased induction of epithelial apoptosis, both changes contributing to compromised barrier function and the opening of the leak pathway induced by $C$. jejuni. In parallel, the recovery experiments with the application of resveratrol revealed a functional improvement of the disturbed epithelial barrier in both models in vitro and in vivo. During treatment with resveratrol, TJ localization of occludin and claudin-5 was fully restored in the paracellular domain of HT-29/B6 cells. Moreover, resveratrol decreased the rate of epithelial apoptosis. These resveratrol-induced molecular and cellular effects would therefore be expected to improve epithelial barrier function, thereby minimizing the so-called leaky gut phenomenon. In conclusion, the induction of the leak pathway by $C$. jejuni and the restoration of barrier function by resveratrol demonstrates its effectiveness as a potential preventive or therapeutic method of mitigating the leaky gut associated with campylobacteriosis.

Keywords: epithelial barrier, mucosal permeability, leak pathway, tight junction, epithelial apoptosis, leak flux, leaky gut model 


\section{INTRODUCTION}

Campylobacter jejuni is a Gram-negative bacterial human pathogen of the gastrointestinal tract. It is a microaerophilic, motile, curvedshaped rod, belonging to the epsilon-proteobacteria group, related to the genera Helicobacter, Wolinella and Aliarcobacter (Vandamme et al., 1991). In animal hosts, mainly in the intestines of domestic and wild poultry, the bacteria are commensals (Shane, 1992). Transmission of $C$. jejuni to humans occurs mostly via undercooked meat or contaminated food (Shane, 1992). After oral uptake of $C$. jejuni by the human host, the bacteria penetrate the intestinal mucus and invade the epithelium in the small and large intestine, leading to diarrhea, gastroenteritis, and fever (Wassenaar and Blaser, 1999).

Campylobacter jejuni is able to actively migrate through the intestinal epithelial layer and thereby causes dysfunction of the epithelial barrier, inducing diarrhea by the leak flux mechanism (Bücker et al., 2018; Butkevych et al., 2020). Hundreds of attempts to find an exotoxin from C. jejuni that could explain its barrierbreaking effects were not successful. The missing evidence for an exotoxinogenic barrier leakage by the bacteria led to the conclusion that the lipooligosaccharides (LOS) and other endotoxins of the bacteria mediate the main pathophysiological events of barrier dysfunction and an exuberant immune response in the mucosal layers (Wassenaar and Blaser, 1999). The major role of LOS in C. jejuni-mediated intestinal pathogenesis was further supported recently by independent findings in humans (Bücker et al., 2018) and in various murine models of campylobacteriosis (reviewed by Mousavi et al., 2020). The downward spiral in the infected intestinal mucosa includes the innate immune activation including subepithelial macrophages and granulocytes (a mucosal cytokine storm), which in turn aggravates epithelial leakage, which leads again to further influx of antigens (Butkevych et al., 2020; Lobo de Sá et al., 2021). The so-called leaky gut phenomenon is the model representation for this cytokine-induced barrier impairment (Hollander, 1999; Schulzke et al., 2006). To describe the pathogenesis of this phenomenon, intestinal biopsies from animals or patients as well as cell culture monolayers interacting with subepithelial cytokine release from lymphocytes, can be used as experimental leaky gut models (Bücker et al., 2014; Bücker et al., 2018).

However, the molecular and structural changes in the $C$. jejuni-infected epithelium include induction of epithelial apoptosis and downregulation of tight junction (TJ) protein expression by genomic regulation of claudin expression, accompanied by a subcellular redistribution of barrier-forming claudins. The direct (bacterial) and indirect (inflammatory) pathological events caused by $C$. jejuni in the epithelium explain infective/inflammatory diarrhea in humans. Proinflammatory activation is the hallmark of infection (Black et al., 1988), in which the release of barrier-affecting cytokines, such as tumor necrosis factor alpha (TNF- $\alpha$ ), interferon gamma $($ IFN- $\gamma$ ), or interleukin-1 beta (IL-1 $\beta$ ) within the intestinal mucosa seems to play a major role in the perpetuation of the diarrhea (Rees et al., 2008; Amasheh et al., 2010; Luettig et al., 2016). Pro-inflammatory cytokines and the bacterium per se target epithelial TJ proteins and trigger epithelial cell death mechanisms (Bücker et al., 2018).

Curcumin from the Turmeric root, Curcuma longa L., is effective against $C$. jejuni infection in vitro as well as in vivo (Lobo de Sá et al., 2019). Resveratrol is another polyphenolic compound and traditional remedy, which we have shown in the present study to possess barrier-restorative properties in different models. In the literature, plant-derived traditional remedies containing resveratrol are broadly described for treatment options of different diseases including inflammatory or cardiovascular diseases (Chudzińska et al., 2020).

The polyphenolic micronutrient resveratrol (3, 5, 4' trihydroxystilbene) is a bioactive compound found in various plants such as wine grapes $(0.1-3.0 \mu \mathrm{g} / \mathrm{g})$, berries $(\sim 0.03 \mu \mathrm{g} / \mathrm{g})$, peanuts $(0.01-2.00 \mu \mathrm{g} / \mathrm{g})$, and in the traditional Asian remedy for inflammatory indications, the Japanese knotweed Reynoutria japonica, which has the highest concentration of resveratrol $(>500 \mu \mathrm{g} / \mathrm{g})$ (Koushki et al., 2018a; Liu et al., 2020). For the preparation of knotweed, recipes from Japan or Korea and in traditional Chinese medicine recommended the use of young raw sprouts as salad or the roots as tincture or powder, to provide sufficient pharmacological concentrations of resveratrol. The anti-inflammatory properties of resveratrol were described in several studies. Improvement of the intestinal epithelial barrier by the molecular effects of resveratrol on TJ stability under inflammatory conditions independently of $C$. jejuni infection have also been reported (Carrasco-Pozo et al., 2013; Ling et al., 2016; Mayangsari and Suzuki, 2018; Cao et al., 2019). The phytopharmaceutical resveratrol is effective in reducing inflammatory parameters such as TNF- $\alpha$ or C-reactive protein (CRP) levels in clinical trials (Koushki et al., 2018b). Effective concentrations of resveratrol are not usually provided by a normal diet (Weiskirchen and Weiskirchen, 2016). Only traditional remedy plants or pharmaceutical preparations provide pharmacological relevant concentrations of resveratrol, but these can cause adverse effects (Weiskirchen and Weiskirchen, 2016; Salehi et al., 2018).

Concerning C. jejuni infections, we have shown recently that resveratrol treatment alleviates intestinal inflammation and macroscopic sequelae of campylobacteriosis in a standardized murine preclinical disease model (Heimesaat et al., 2020). To determine mechanisms underlying the beneficial effects of resveratrol in the treatment of campylobacteriosis, we performed experiments in different models. Human intestinal HT-29/B6 cell monolayers in conjunction with inflammatory conditions were used as in vitro model of the leaky gut, and an in vivo hyperacute animal model was used to study intestinal leakiness and inflammation. The Campylobacter-infected secondary abiotic IL-10 $0^{-/-}$mouse model was used to confirm the mechanistic effects observed in vitro.

\section{MATERIALS AND METHODS}

\section{Bioinformatics on RNA-Seq Data}

Based on a previously performed RNA-Sequencing (RNA-Seq) analysis with subsequent Ingenuity Pathway Analysis (IPA; 
Qiagen, Redwood, CA, United States) of human colonic mucosa from four C. jejuni infected patients and six control patients, several compounds were identified as potential inhibitory or therapeutic agents in campylobacteriosis. Procedures of RNA extraction, RNA sequencing, and Ingenuity Pathway Analysis were performed previously (Bücker et al., 2018). The sequence data set for the bioinformatics prediction is available under NCBI Gene Expression Omnibus (GEO ID GSE88710). In the IPA prediction for the potential compound resveratrol, the Upstream regulator analysis software tool was used. The detailed information on the calculation method and statistics is available on Ingenuity-Qiagen websites. In brief, Upstream regulator analysis is based on prior knowledge from the literature of expected effects between transcriptional regulators and their target genes. The analysis examines how many known targets of each transcription regulator are regulated in the RNA dataset of the patients. For each potential transcriptional regulator, two statistical measures - an overlap $p$-value and an activation $Z$-score - are computed. The overlap $p$-value predicts likely upstream regulators based on significant overlap between dataset genes and known targets regulated by a transcription regulator or upstream regulator. The activation $Z$-score is used to infer likely activation states of upstream regulators based on comparison with a model that assigns random regulation directions. The purpose of the overlap $p$-value is to identify transcriptional regulators that are able to explain observed gene expression changes. The overlap $p$-value determines whether there is a statistically significant overlap between the dataset genes and the genes that are regulated by an upstream transcription regulator. It is calculated using Fisher's Exact Test, and significance is generally attributed to $p$-values $<0.01$. Since the regulation direction ("activating" or "inhibiting") of a relationship is not taken into account for the computation of overlap $p$-values, the underlying network also includes findings without associated directional attribute, such as protein-DNA promoter binding. The primary purpose of the activation $Z$-score is to infer the activation states of predicted upstream transcription regulators. The basis for inference are the relationships in the molecular network that represent experimentally observed gene expression or transcription events, and are associated with a literature-derived regulation direction, which can be either "activating" or "inhibiting". The statistical approach defining a quantity ( $Z$-score) determines whether an upstream transcription regulator has significantly more "activated" predictions than "inhibited" predictions $(Z>0)$ or vice versa $(Z<0)$. The definition of upstream transcriptional regulator in this analysis is broad, namely any molecule that can affect the expression of other molecules, which means that upstream regulators can be almost any type of molecule, from transcription factors to microRNAs, kinases, compounds or drugs.

\section{Bacterial Cultivation}

The reference strain Campylobacter jejuni wildtype 81-176 was pre-cultured on blood agar plates at $37^{\circ} \mathrm{C}$ in plastic jars with CampyGen gas packs from Oxoid (Thermo Scientific, Waltham, MA, United States) to generate a microaerobic atmosphere. After $48 \mathrm{~h}$ of incubation bacterial colonies were transferred into
Mueller Hinton broth and incubated with shaking (200 rpm) at $37^{\circ} \mathrm{C}$ for $2 \mathrm{~h}$ under microaerobic conditions. Bacteria were centrifuged $\left(5000 \times g\right.$, for $2 \mathrm{~min}$, at $\left.10^{\circ} \mathrm{C}\right)$, resuspended in phosphate buffered saline (PBS; Gibco, Carlsbad, CA, United States) and adjusted to an optical density $\mathrm{OD}_{600}$ of one in the cell culture medium for the application of a defined multiplicity of infection (MOI) to the epithelial cell monolayers.

\section{Human Cell Culture Model}

HT-29/B6-GR/MR cells (Bergann et al., 2009) were cultivated in $25 \mathrm{~cm}^{2}$ culture flask in RPMI 1640 culture medium (Sigma Aldrich, St. Louis, MO, United States) supplemented with $10 \%$ fetal calf serum (FCS, Gibco, Carlsbad, CA, United States), 1\% penicillin/ streptomycin (Corning, Wiesbaden, Germany), $300 \mu \mathrm{g} / \mathrm{ml}$ G418 BC (Invitrogen, Carlsbad, CA, USA), and $200 \mu \mathrm{L} / \mathrm{ml}$ hygromycin B (Biochrom $\mathrm{GmbH}$, Berlin, Germany). Every seven days cells were passaged and then seeded on Millicell PCF filter membranes (Merck Millipore, Billerica, MA, United States) with a pore size of $3 \mu \mathrm{m}$, where they formed confluent epithelial monolayers. Six days after seeding the cells were washed twice with antibiotic-free cell culture medium supplemented with 10\% heat-inactivated FCS. Seven days after seeding the cells reached transepithelial electrical resistances (TER values) of $600-900 \Omega \cdot \mathrm{cm}^{2}$.The cells were treated with $100 \mu \mathrm{M}$ trans-resveratrol ( $\geq 99 \%$ purity (HPLC), 3,4',5Trihydroxy-trans-stilben, 5-[(1E)-2-(4-Hydroxyphenyl)-ethenyl]1,3-dihydroxybenzol, Sigma Aldrich, St. Louis, MO, United States) from the apical compartment $2 \mathrm{~h}$ before apical infection with C. jejuni (MOI 100).

\section{Electrophysiological Measurements of Barrier Function and Permeability Measurements}

Transepithelial electrical resistance (TER) of the cell monolayers was measured under sterile conditions with chopstick electrodes (STX2, World Precision Instruments, Sarasota, FL, United States) and a volt-ohm meter (Clinical Physiology, Charité-Universitätsmedizin, Berlin). Measured TER values were corrected with the TER of an empty filter support and calculated on the surface area in square centimeters with the effective growth area of $0.6 \mathrm{~cm}^{2}$. In parallel, small molecule permeability was determined by measuring fluxes of the tracer fluorescein (332 Da, Sigma Aldrich, St. Louis, MO, United States) in 12-well plates. Fluorescent medium samples were taken from the basal compartment for $1 \mathrm{~h}$ (every $15 \mathrm{~min}$ ), and the samples were subsequently analyzed by spectrophotometry (Tecan $\mathrm{GmbH}$, Maennendorf, Switzerland). Permeability for fluorescein was calculated from the flux divided by the concentration difference.

\section{Cytokine-Stimulated in vitro Model}

Epithelial cells were treated for $24 \mathrm{~h}$ with different concentrations and combinations of IFN- $\gamma$, TNF- $\alpha$, and IL- $1 \beta$ (recombinant human cytokines, PeproTech, Rocky Hill, NJ, United States). Cytokines were added to the basolateral compartment. The next day, fluorescein (332 Da) flux measurements were performed in the 12 -well plates for $1 \mathrm{~h}$ (every $15 \mathrm{~min}$ ). 


\section{Campylobacter-Infection and Resveratrol Treatment of the Colitis Mouse Model}

Three-week-old IL-10 ${ }^{-/-}$mice with a C57BL/6j background were treated for eight weeks with an antibiotic cocktail containing ampicillin plus sulbactam $(1 \mathrm{~g} / \mathrm{L} ; \mathrm{Dr}$ Friedrich Eberth Arzneimittel, Ursensollen, Germany), ciprofloxacin (200 mg/ L; Fresenius Kabi, Bad Homburg, Germany), imipenem (250 mg/L; Fresenius Kabi, Bad Homburg, Germany), metronidazole ( $1 \mathrm{~g} / \mathrm{L}$; Fresenius, Bad Homburg, Germany), and vancomycin $(500 \mathrm{mg} / \mathrm{L}$; Hikma Pharmaceuticals, London, United Kingdom) in autoclaved drinking water ad libitum to remove the commensal intestinal microbiota. Generation, housing, infection, and treatment of the IL$10^{-/-}$mice were carried out under specific pathogen-free conditions in the animal facility of the Forschungseinrichtung für Experimentelle Medizin of the Charité-Universitätsmedizin Berlin. Two days before infection, treatment was started with trans-resveratrol $(0.3 \mathrm{mg} / \mathrm{ml}$ [ $1.3 \mathrm{mM}]$ resveratrol dissolved in $2 \%$ carboxymethyl-cellulose) in drinking water provided ad libitum. Mice were infected on day 0 and day 1 by oral gavage with $10^{9}$ colony forming units (CFU) of $C$. jejuni (treatment group, named "resveratrol $+C$. jejuni"). The control group was $C$. jejuni-infected but received only drinking water containing the carrier-solution (placebo control, named "C. jejuni"). A small control group of naïve healthy mice was used for baseline measurements (named "control"). Mice were sacrificed at day 6 after infection and intestinal specimens were removed for fluorescein permeability or cytokine measurements. For cytokine assessment in organs, colon and mesenteric lymph nodes specimens were cultured in 24-well plates in serum-free RPMI 1640 culture medium supplemented with penicillin and streptomycin for $18 \mathrm{~h}$ at $37^{\circ} \mathrm{C}$. IFN- $\gamma$ and TNF- $\alpha$ concentrations were determined in the cell culture supernatant with the Mouse Inflammation Cytometric Bead Array (CBA; BD Biosciences, Heidelberg, Germany) using a FACSCanto II flow cytometer (BD Biosciences, Heidelberg, Germany).

\section{Ethical Statements}

Animal experiments were carried out in accordance with the German Animal Protection Act and the ARRIVE guidelines. The study was approved by the ethics committee for animal welfare in Berlin, Landesamt für Gesundheit und Soziales (LAGeSo Berlin) under the LAGeSo approval number G0104/19.

\section{Western Blotting}

Tight junction protein expression changes were analyzed by Western blotting. Proteins from cell culture experiments were lyzed using

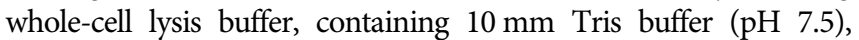
$150 \mathrm{~mm} \mathrm{NaCl}, 0.5 \%$ Triton X-100, 1\% SDS, and one tablet Complete Protease Inhibitor Cocktail (Roche AG, Mannheim, Germany). After lysis, cells were scraped carefully from the filter supports, added to a reaction tube, incubated on ice for 30-60 min, and centrifuged for $30 \mathrm{~min}$ at $15000 \mathrm{x} \mathrm{g}$ and $4^{\circ} \mathrm{C}$. After sonication and a further centrifugation step, protein quantification followed with the Pierce BCA kit (Thermo Scientific, Waltham, MA, United States). Electrophoresis was performed in $12.5 \%$ polyacrylamide gels and transferred to nitrocellulose membranes. After blocking for $2 \mathrm{~h}$ in 1\% Polyvinylpyrrolidone (PVP40; Sigma Aldrich, St. Louis, MO, United States) and 0.05\% Polysorbate 20 (Tween20; Thermo Scientific, Waltham, MA, United States) primary antibodies raised against human occludin, claudin-2 (1:1000; Sigma Aldrich, St. Louis, MO, United States), claudin- $1,-4,-5,-7,-8$ (1: 1000; Invitrogen, Carlsbad, CA, United States), and $\beta$-actin (1:10000; Sigma Aldrich, St. Louis, MO, United States) as loading control, were incubated slewing at $4^{\circ} \mathrm{C}$ overnight. Next day, membranes were washed with TBST buffer and incubated with secondary peroxidaseconjugated antibodies goat anti-rabbit IgG or goat anti-mouse IgG (Jackson ImmunoResearch, Ely, United Kingdom) in TBST supplemented with $1 \%$ milk powder (Sigma Aldrich, St. Louis, $\mathrm{MO}$, United States) for $2 \mathrm{~h}$ at room temperature. For protein detection, membranes were incubated for $2-5 \mathrm{~min}$ in SuperSignal West Pico PLUS Stable Peroxide Solution (Thermo Scientific, Waltham, MA, United States) and visualized using a Fusion FX7 imaging system (Vilber Lourmat Deutschland GmbH, Eberhardzell, Germany). Image Studio Lite version 5.2 was used for densitometric analysis.

\section{Immunofluorescence Microscopy of Tight Junction Proteins and Apoptosis}

Cells grown on filter supports were rinsed twice with PBS and fixed with $2 \%$ paraformaldehyde (Electron Microscopy Sciences, Hatfield, PA, United States) for $30 \mathrm{~min}$ at room temperature. After permeabilization for $7 \mathrm{~min}$ with $0.5 \%$ Triton X-100 (Sigma Aldrich, St. Louis, MO, United States), cells were blocked $10 \mathrm{~min}$ at room temperature with blocking solution containing $5 \%$ goat serum (Gibson, Carlsbad, CA, United States), 0.05\% Triton X100 , and $1 \%$ bovine serum albumin (BSA; Sigma Aldrich, St. Louis, MO, United States). Afterwards, cells were incubated for $1 \mathrm{~h}$ at $37^{\circ} \mathrm{C}$ with primary antibodies anti-occludin (1:100; Sigma Aldrich, St. Louis, MO, United States), anti-claudin-5 (1:100; Invitrogen, Carlsbad, CA, United States), and anti-zonula occludens protein-1 (ZO-1, 1:100; BD Biosciences, Franklin Lakes, NJ, United States) followed by the secondary anti-rabbit or anti-mouse antibody conjugated to Alexa-Fluor 488 or 594 (1: 500; Invitrogen, Carlsbad, CA, United States) for $1 \mathrm{~h}$ at $37^{\circ} \mathrm{C}$. For apoptosis detection, cells were stained with the TUNEL kit (In situ Cell Death Detection Kit, Roche AG, Mannheim, Germany) according to manufacturer's instructions. Nuclei were stained with 4,6-diamidino-2-phenylindole (DAPI; 1:1000; Roche AG, Basel, Switzerland). Visualization was performed by confocal laser-scanning microscopy (CLSM; Zeiss LSM780, Jena, Germany).

\section{Statistics}

All data are expressed as mean values \pm standard error of the mean (SEM). Statistical analysis was performed using GraphPad Prism version 8.0. For comparison of two groups, Student's $t$-test and for comparison of more than two groups one-way ANOVA with Bonferroni adjustment for multiple comparisons was used. Data that were not normally distributed were analyzed using the 
Mann Whitney $U$-test. A value $p<0.05$ was considered to be statistically significant.

\section{RESULTS}

\section{Bioinformatics Prediction for the Suitability of Resveratrol}

In a bioinformatics calculation from our existing dataset of a differential RNA expression profile in C. jejuni-infected human colonic mucosa, we searched putative suitable compounds for the treatment of campylobacteriosis. In the Ingenuity Pathway Analysis, resveratrol was found to show an inhibited pathway profile in the Upstream regulator analysis with $1.09 \mathrm{E}^{-5} p$-value of overlap and an activation $Z$-score of -2.8 (negative score, pointing to inhibited pathways/downstream targets), indicating that the presence of resveratrol could counter-regulate the $C$. jejuniinduced changes. As an Upstream regulator, resveratrol shows comparable values for inhibited pathways like the nutraceutical polyphenol curcumin with a $p$-value of $2.06 \mathrm{E}^{-5}$ or quercetin with a $p$-value $1.95 \mathrm{E}^{-5}$. By contrast, activated pathways with positive $\mathrm{Z}$-scores and comparable $p$-values were calculated for proinflammatory cytokines, e.g. IL-17; overlap $p$-value $1.71 \mathrm{E}^{-5}$, $Z$-score +3.7 (with activated pathways/downstream targets). The cytokines with the highest significance for activated pathways IFN$\gamma$ and TNF- $\alpha$ showed activation $Z$-scores of +9.6 and +9.0 respectively, and both were considered as the major cytokine pathways in campylobacteriosis, as reported previously (Bücker et al., 2018). Sequencing data are deposited in NCBI's Gene Expression Omnibus; GEO ID GSE88710.

\section{Epithelial Barrier Dysfunction After Campylobacter jejuni Infection in HT-29/B6 Monolayers Was Rescued by Resveratrol}

The induction of epithelial barrier impairment by $C$. jejuni has already been described (Bücker et al., 2018). The inhibitory effect of resveratrol on the $C$. jejuni-induced barrier dysfunction was tested here for the first time in vitro. In our infection experiments with C. jejuni, pharmacological relevant concentrations of resveratrol were chosen to investigate the actions of resveratrol on the intestinal epithelium without directly inhibiting the bacteria. Administration of $100 \mu \mathrm{M}$ resveratrol $(=23 \mu \mathrm{g} / \mathrm{ml})$ alone to HT-29/B6 monolayers did not induce any changes in TER over two days of incubation (Figure 1A). Infection of HT29/B6 monolayers with C. jejuni from the apical side revealed a drop in TER to $43 \pm 5 \%$ of the initial value after $48 \mathrm{~h}$ (Figure 1A). This barrier-breaking effect was prevented by the addition of resveratrol (Figure 1A).

\section{Paracellular Flux Marker Fluorescein Showed Increased Permeability After Campylobacter jejuni Infection and its Reversal by Resveratrol Treatment}

The TER represents the permeability for ions, whereas the paracellular leakiness or tightness of an epithelium towards
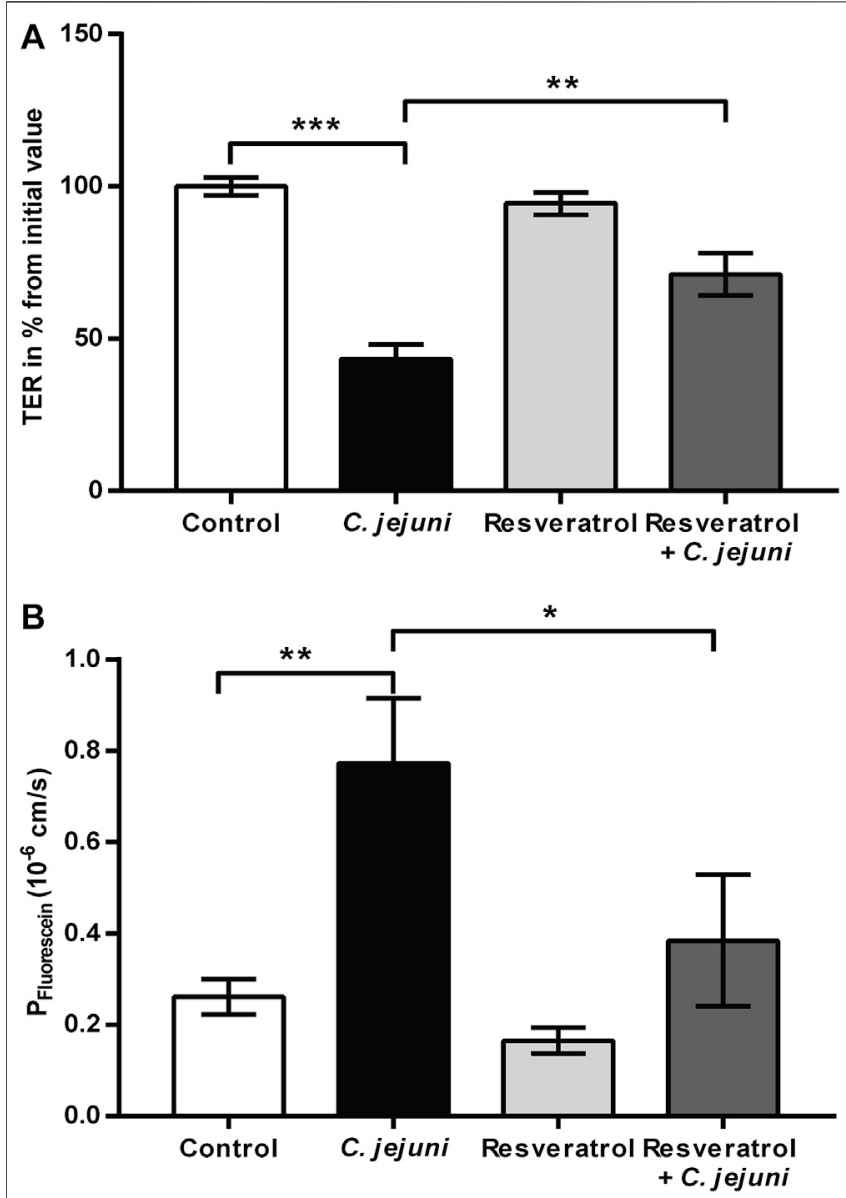

FIGURE 1 | Epithelial barrier function and macromolecule flux after Campylobacter jejuni infection in epithelial cell monolayers and after treatment with resveratrol. Human intestinal epithelial HT-29/B6 cells were treated with $100 \mu \mathrm{M}$ of resveratrol from apical and infected apically with $C$. jejuni (multiplicity of infection; MOI 100). Transepithelial electrical resistance (TER) was measured with chopstick electrodes $48 \mathrm{~h}$ after the infection (A) Resveratrol inhibits the $C$. jejuni-induced decrease in TER in vitro. Control values after $48 \mathrm{~h}$ were set at $100 \%\left(\mathrm{n}=9-10,{ }^{\star \star} p<0.01\right.$, one-way ANOVA with Bonferroni correction). (B) Resveratrol prevents $C$. jejuni-induced increase in permeability to fluorescein (332 Da) in vitro $\left(n=10,{ }^{*} p<0.05,{ }^{* *} p<\right.$ 0.01, one-way ANOVA with Bonferroni correction).

larger molecules is represented by marker fluxes. In order to characterize the barrier defect by $C$. jejuni on the paracellular pathway, we used the flux marker fluorescein (332 Da). Flux measurements between the apical and basolateral side of HT-29/ B6 monolayers revealed an increase in permeability for fluorescein in C. jejuni-infected monolayers, whereas the resveratrol-treated and infected monolayers showed reduced fluorescein permeability in comparison to the C. jejuniinfected group. The resveratrol-treated C. jejuni-infected cells showed comparable results to the uninfected untreated controls $(p>0.05)$ (Figure 1B). Thus, the TER and fluorescein measurements indicate that resveratrol prevented the disturbance in epithelial barrier function induced by $C$. jejuni. 

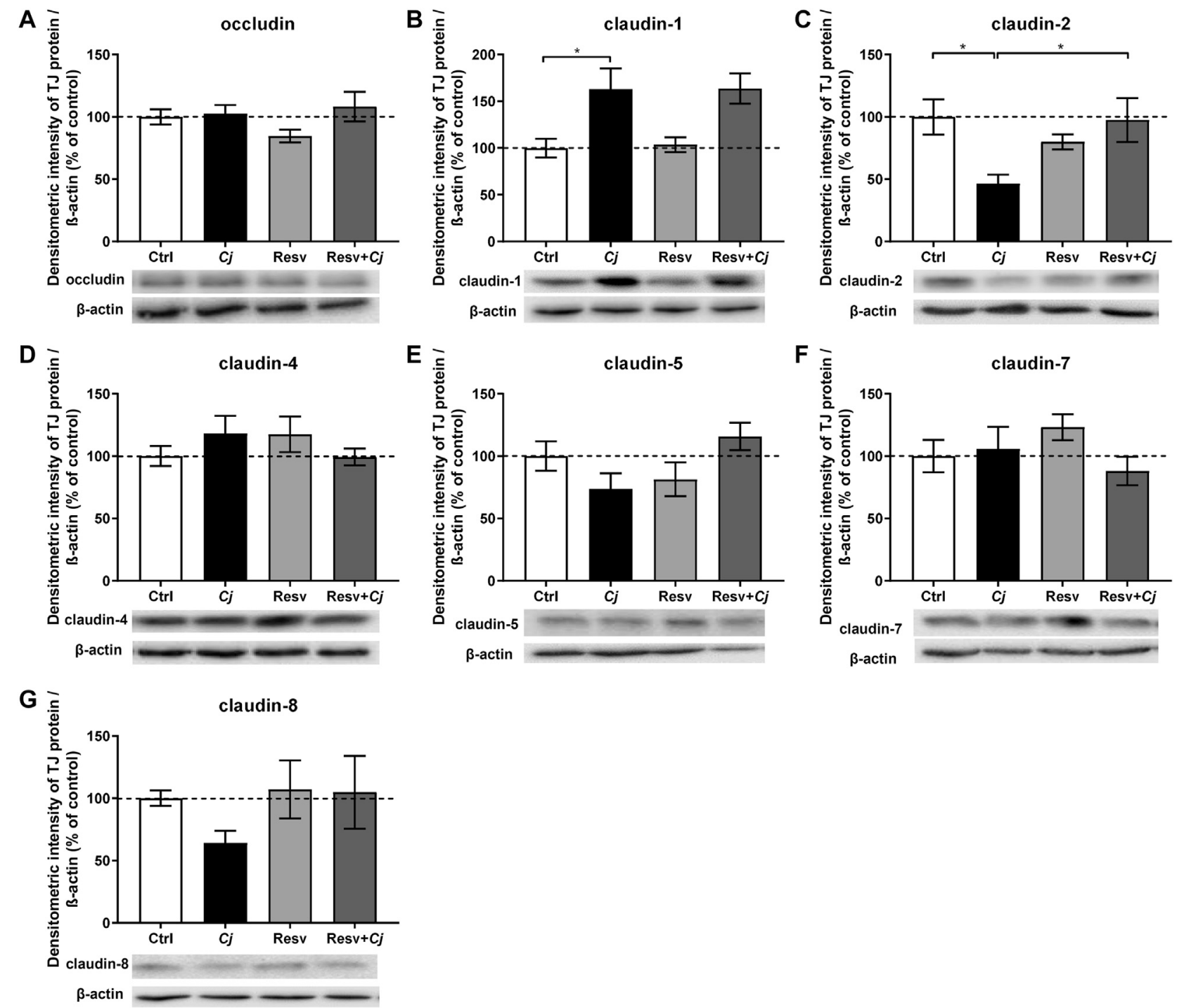

FIGURE 2 | Expression of tight junction proteins after Campylobacter jejuni infection and resveratrol treatment. Epithelial tight junction protein expression changes under C. jejuni (Cj) infection and resveratrol (Resv) treatment in vitro. Proteins from HT-29/B6-GR/MR cell monolayers were isolated after $48 \mathrm{~h}$ C. jejuni infection with or without $100 \mu \mathrm{M}$ resveratrol treatment for protein expression analysis by Western blotting (A) occludin (B) claudin-1 (C) claudin-2 (D) claudin-4 (E) claudin-5 (F) claudin7 , and (G) claudin-8 expression were quantified by densitometric analysis of Western blots, normalized to $\beta$-actin. Untreated uninfected cells were set as controls (Ctrl) at $100 \%\left(n=5-10,{ }^{*} p<0.05\right.$, one-way ANOVA with Bonferroni correction).

\section{Tight Junction Expression and its Subcellular Distribution Change During Campylobacter jejuni Infection and Resveratrol Treatment in HT-29/B6 Monolayers}

The molecular basis of an impaired leak pathway is often based on the expression and/or distribution of TJ proteins. To detect changes in TJ protein expression in HT-29/B6 cell monolayers, we used Western blotting with antibodies raised against occludin and claudins (Figure 2). Expression of occludin (Figure 2A), claudin-4 (Figure 2D) and claudin-7 (Figure 2F) in HT-29/B6 monolayers was unaffected by $C$. jejuni infection or resveratrol treatment.
Although the expression level of claudin-5 (Figure 2E) and claudin-8 (Figure 2G) showed a tendency towards a reduction after infection and to be reversed by resveratrol treatment, this did not reach statistical significance. Moreover, infection did not appear to induce the cleavage of occludin. However, claudin-2 protein expression was decreased and fully restored to control levels by resveratrol (Figure 2C), while claudin-1 expression was increased after infection and was unchanged by resveratrol (Figure 2B).

To determine the effects of $C$. jejuni and resveratrol on the localization of TJ proteins, we performed fluorescent immunostaining for confocal laser-scanning microscopy (CLSM) and analyzed the micrographs using the intensity plot function of the CLSM software. After infection with C. jejuni the TJ proteins, 

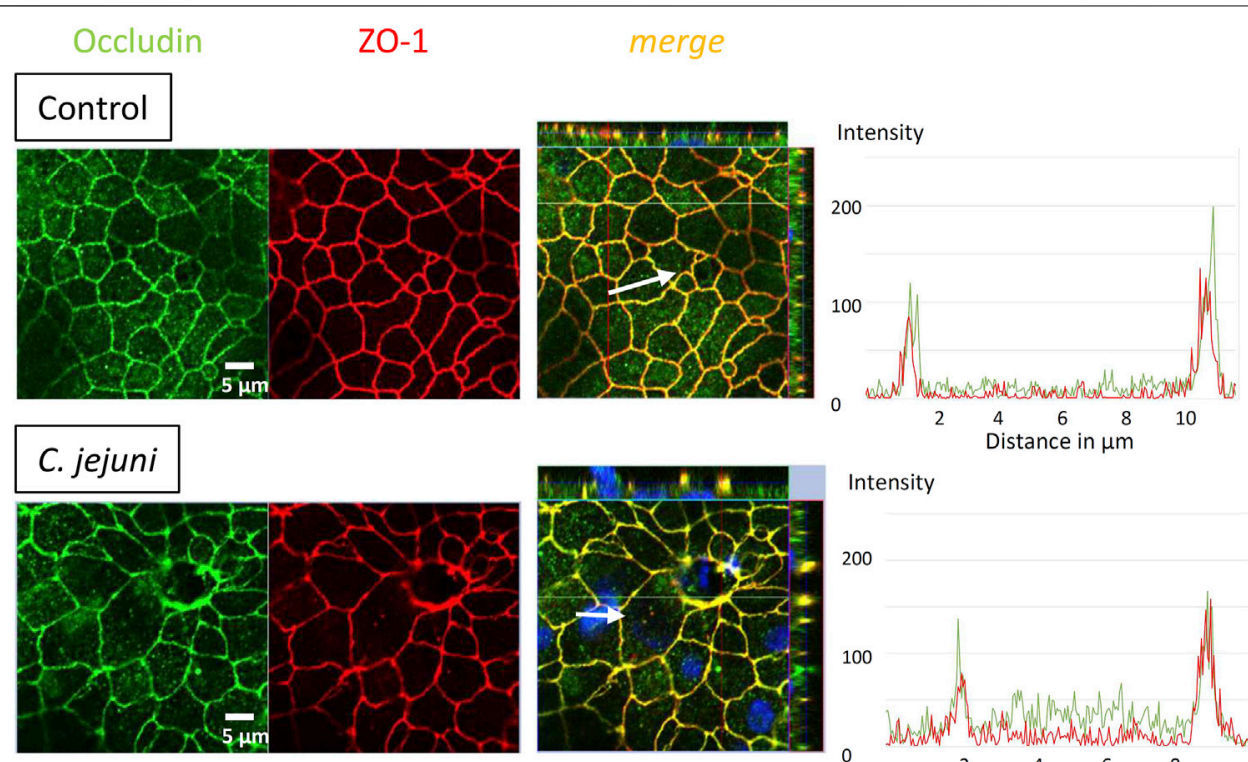

Intensity

200

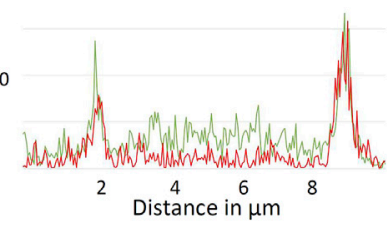

Resveratrol $+C$. jejuni
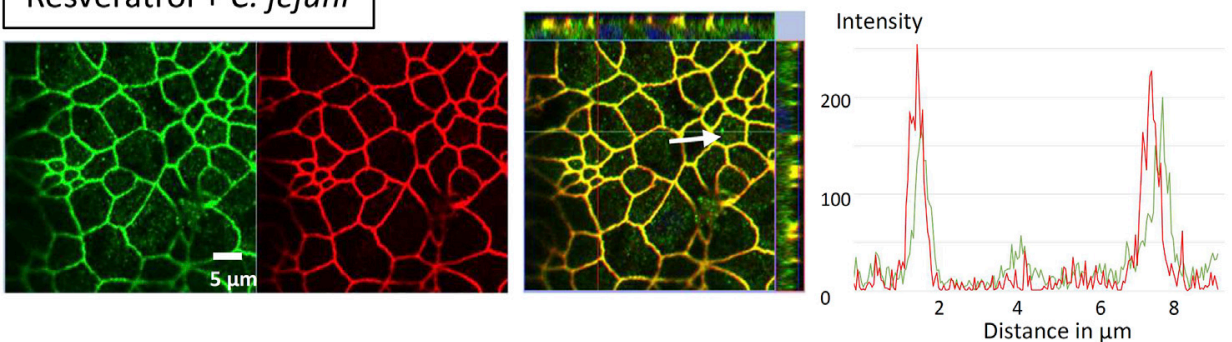

FIGURE 3 | Subcellular tight junction protein distribution of occludin under Campylobacter jejuni infection and resveratrol treatment. Resveratrol mitigates C. jejuniinduced re-distribution of occludin. HT-29/B6-GR/MR cells were treated with resveratrol and infected with C. jejuni from apical. $48 \mathrm{~h}$ post-infection monolayers grown on filter inserts were stained with antibodies against occludin (green) and zonula occludens protein-1 (ZO-1; red). Nuclei were stained with 4,6-diamidino-2-phenylindole (DAPI) in blue (bar $5 \mu \mathrm{m})$. Intensity plots point to signals of the TJ proteins indicated with white arrows in the merge pictures. The intensity signals appear highest in the TJ domain with a proper co-localization under control conditions and vary under infection and treatment conditions along the white arrows over single cells.

occludin and claudin-5 were redistributed off the TJ domain of the epithelial cells (Figures 3, 4). Occludin signals were found to be intracellular and no longer co-localized with ZO-1 in the TJ strands (Figure 3). Even more pronounced was the redistribution of claudin-5 off the TJ domain and the appearance of intracellular claudin-5 signals (Figure 4). In Figures 3, 4, intensity plots point to increased intracellular signals of occludin or claudin- 5 in response to infection, which were diminished after resveratrol treatment. The change in the expression profiles of claudin -1 and -2 (and -5 and -8 by trend), and the subcellular signals of occludin and claudin- 5 detected by CLSM, resembles a restitution reaction of the epithelium as described previously during $C$. jejuni infection and also observed during the induction of apoptosis (Bojarski et al., 2004; Amasheh et al., 2010; Rosenthal et al., 2017; Bücker et al., 2018; Butkevych et al., 2020).

\section{Induction of Epithelial Apoptosis by Campylobacter jejuni and its Recovery by Resveratrol}

In addition to TJ disruption during $C$. jejuni infection, the induction of epithelial apoptosis represents a pathomechanism which is relevant to the disruption of barrier function. C. jejuni induces epithelial apoptosis via cleavage of caspase-3 (Bücker et al., 2018; Lobo de Sá et al., 2019; Mousavi et al., 2019; Butkevych et al., 2020). We therefore studied the possible therapeutic benefit of resveratrol on the induction of apoptosis. TUNEL staining was applied and visualized using CLSM. C. jejuni infection increased the apoptotic ratio in the HT29/B6 cell monolayers 3-fold in comparison to controls. Resveratrol treatment of the $C$. jejuni infected monolayers reduced the apoptotic ratio to the level in controls ( $p>0.05$; Figure 5). Thus, the ability of resveratrol to prevent epithelial cell death as well as restore TJ protein localization explains its efficacy in preventing the epithelial barrier defects induced by $C$. jejuni infection.

\section{In vitro Effects of Pro-inflammatory Cytokines and Their Prevention by Resveratrol}

We analyzed the effect of resveratrol on cytokine-induced barrier disruption using a cell model of the leaky gut. We mimicked subepithelial cytokine release in the epithelial cell model by adding 

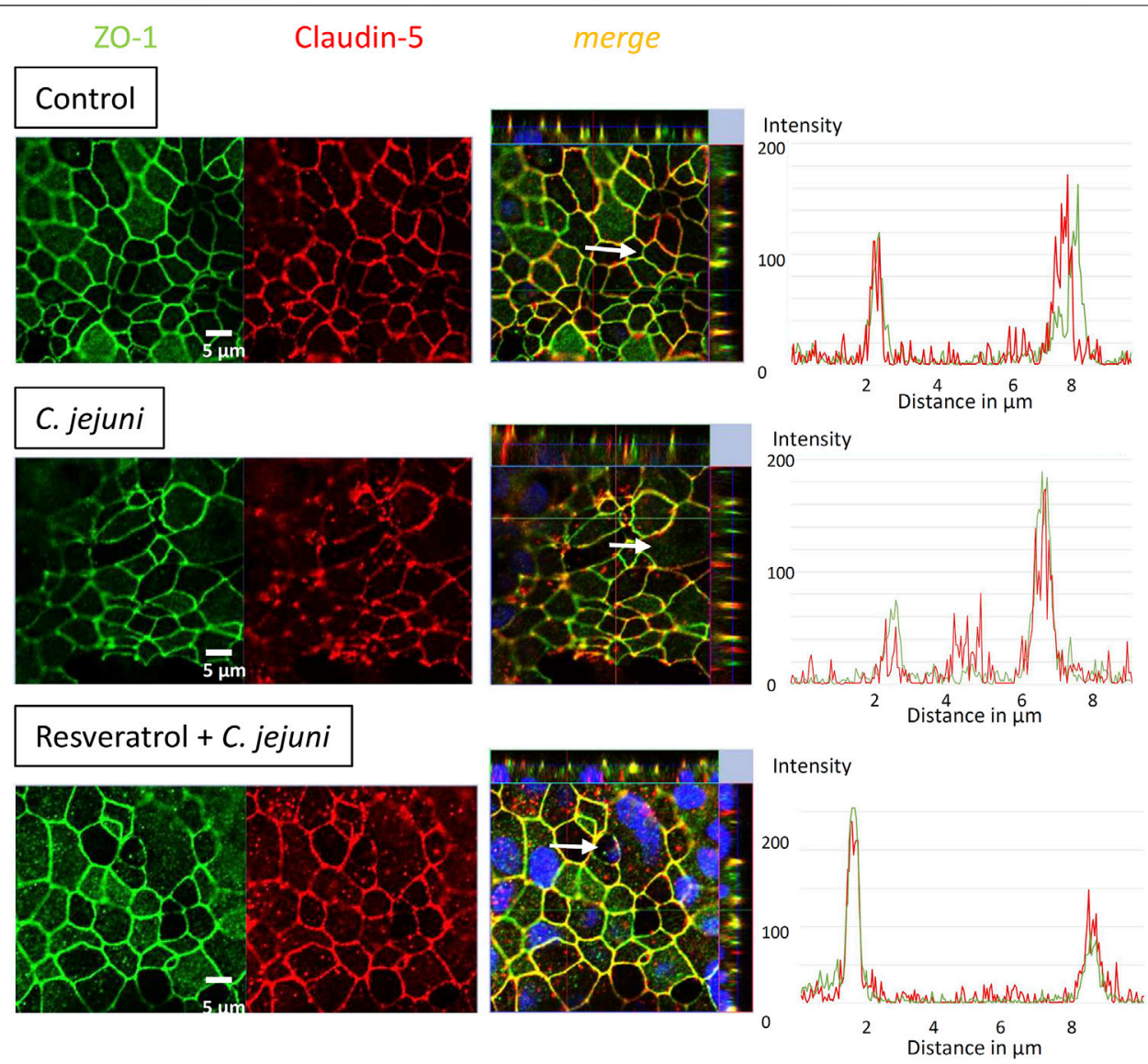

FIGURE 4 | Subcellular tight junction protein distribution of claudin-5 under Campylobacter jejuni infection and resveratrol treatment. Resveratrol mitigates $C$. jejuniinduced re-distribution of claudin-5. HT-29/B6-GR/MR cells were treated with resveratrol and infected with C. jejuni from apical. $48 \mathrm{~h}$ post-infection monolayers were stained with antibodies against claudin- 5 (red) and ZO-1 (green). Nuclei were stained blue with DAPI (bar $5 \mu \mathrm{m})$. Intensity plots point to signals of the TJ proteins along the white arrows in the merge pictures. The intensity signals appear highest in the TJ domain with a proper co-localization under control conditions and vary under infection and treatment conditions with the white arrows depicted over single cells.

the major cytokines found in campylobacteriosis, namely of IFN- $\gamma$, TNF- $\alpha$ and IL-1 $\beta$. This model resembles the leaky gut situation, where stimulated subepithelial immune cells release these cytokines. When the single cytokine $50 \mathrm{mg} / \mathrm{ml} \mathrm{IFN}-\gamma$ was added, fluorescein permeability increased and this effect was attenuated by resveratrol (Figure 6A). By contrast, addition of IFN- $\gamma$, TNF- $\alpha$ and IL- $1 \beta$ at high doses induced an increase in paracellular permeability for fluorescein, but no recovery was obtained with $100 \mu \mathrm{M}$ resveratrol (Figure 6B). These experiments indicate that the effects of cytokines at submaximal but not at maximal concentrations are counteracted by resveratrol.

\section{In vivo Confirmation of Barrier Impairment of the Paracellular Pathway in the $C$. jejuni-Infected Mouse Model and its Prevention by Resveratrol}

To study the effectiveness and the mechanism of the effect of resveratrol on barrier function seen in vitro and in vivo, the $C$. jejuni-infected secondary abiotic IL- $10^{-/-}$mouse model was used. Estimation of the intestinal epithelial leakiness was evaluated, by measuring fluxes of the paracellular flux marker fluorescein across colon from Campylobacter-infected mice mounted in Ussing chambers. Paracellular permeability for fluorescein was higher in colon mucosae of $C$. jejuni-infected mice compared with resveratrol-treated mice at day 6 post-infection $(p<0.05$; Figure 7A), indicating that resveratrol treatment rescues colonic epithelial barrier function following $C$. jejuni infection.

Since mucosal cytokine release could be counter-regulated by resveratrol, we also assessed cytokine production during infection, in our mouse model. The concentrations of IFN- $\gamma$ and TNF- $\alpha$ were elevated in colon mucosae at day 6 postinfection, but did not differ between the C. jejuni-infected placebo and the resveratrol-treated groups (Figures $7 \mathbf{B}, \mathbf{C}$ ). The same was true of cytokine levels in the mesenteric lymph nodes (Figures 7D,E). Thus, in keeping with our in vitro results, the anti-Campylobacter effect of resveratrol seems to be based more on improving barrier function at the epithelial level than by attenuating cytokine release. 


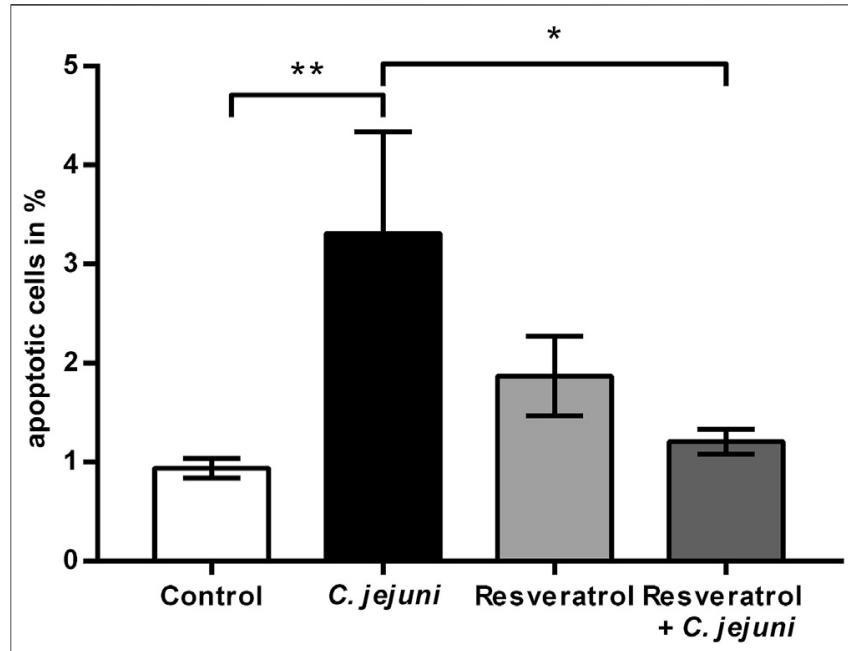

FIGURE 5 | Apoptosis induction as barrier-relevant event and protection by resveratrol. Campylobacter jejuni-induced apoptosis was inhibited by resveratrol. HT-29/B6-GR/MR cells treated with $100 \mu \mathrm{M}$ resveratrol were infected from the apical side with $C$. jejuni. $48 \mathrm{~h}$ post-infection cells were stained with TUNEL reagent to visualize apoptotic cells. 4,6-diamidino-2phenylindole (DAPI) was used for staining of the nuclei. Apoptotic cells were counted in every filter in six different low power fields (approximately 500 cells) to calculate the apoptotic ratio $\left(\mathrm{n}=6,{ }^{*} p<0.05,{ }^{\star *} p<0.01\right.$, one-way ANOVA with Bonferroni correction).

To comply with the guidelines of the $3 \mathrm{R}$ principles to reduce the number of animal experiments, we used animal biopsies and experimental in vivo data from an ongoing study in collaboration (Heimesaat et al., 2020).

\section{DISCUSSION}

\section{Resveratrol - A Valuable Compound in Campylobacter jejuni Infection}

Based on the functional effects of resveratrol reported in the literature as well as on our bioinformatics prediction, this natural compound was deemed to be effective in antagonizing the C. jejuni-induced changes in host epithelial cells. Direct antimicrobial effects on different pathogenic bacteria, including Campylobacter, have been reported at higher concentrations of resveratrol, but not at lower concentrations (minimum inhibitory concentration (MIC) of $300 \mu \mathrm{g} / \mathrm{ml}$ ) (Klančnik et al., 2017). In our in vitro assays, the concentration of resveratrol required to block $C$. jejuniinduced barrier defects was $100 \mu \mathrm{M}(23 \mu \mathrm{g} / \mathrm{ml})$, which was lower than the MIC required to act directly against $C$. jejuni. Likewise, the resveratrol concentration used for the in vivo experiments was close to, but lower than, the MIC of C. jejuni 81-176 with $456.5 \mu \mathrm{g} / \mathrm{ml}$ (Heimesaat et al., 2020). Thus, the protective effects of resveratrol appear to be targeted to the host response rather than to a direct anti-bacterial effect in the infected animals (Heimesaat et al., 2020).

In C. jejuni-infected patients, profound intestinal epithelial barrier dysfunction together with inflammation of the colonic mucosa are prominent features of this diarrheal disease, which is characterized by watery or bloody diarrhea, often accompanied by fever and elevated CRP values (Nielsen et al., 2012; Bücker et al., 2018). The main diarrheal mechanism in C. jejuni infection is based on the leak flux type of diarrhea, in which water and solutes traverse the leaky epithelium towards the intestinal lumen (Bücker et al., 2018). Concomitant with this change in fluid and electrolyte transport, the leaky gut facilitates antigen influx via the dysfunctional epithelial barrier, which results in an accelerated immune response and further disruption of barrier function (a self-sustaining feedback loop).

Underlying our hypothesis and planned approach for treating and preventing campylobacteriosis and its sequelae, such as irritable bowel syndrome (IBS), reactive arthritis and the Guillain-Barré syndrome, is the idea that a compound might strengthen the epithelial barrier, thereby preventing intestinal leakiness and antigen entry from the lumen. In our in vitro experiments, resveratrol restored
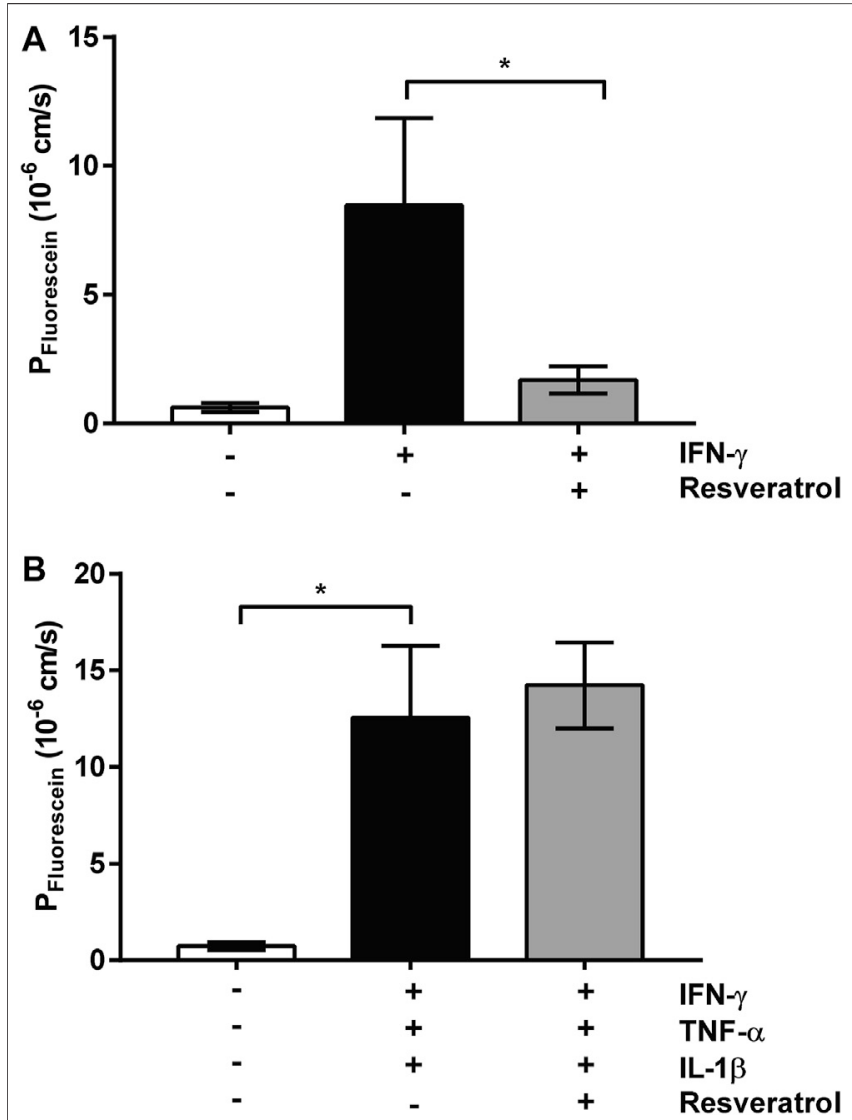

FIGURE 6 | In vitro stimulation model with cytokines and resveratrol. Cytokine-induced barrier dysfunction from the subepithelial compartment of HT-29/B6-GR/MR cell monolayers. Resveratrol treatment was performed from the apical side $2 \mathrm{~h}$ before cytokine addition. Fluorescein permeability displays barrier-breaking effects for (A) $50 \mathrm{ng} / \mathrm{ml}$ IFN- $\gamma$ and (B) high-dose cytokine cocktail containing $100 \mathrm{U} / \mathrm{mL}$ TNF- $\alpha$, and $100 \mathrm{ng} / \mathrm{ml} \mathrm{IL}-$ $1 \beta, 50 \mathrm{ng} / \mathrm{ml}$ IFN- $\gamma\left(n=3-8,{ }^{\star} p<0.05,{ }^{\star \star \star} p<0.001\right.$, one-way ANOVA with Bonferroni correction). 

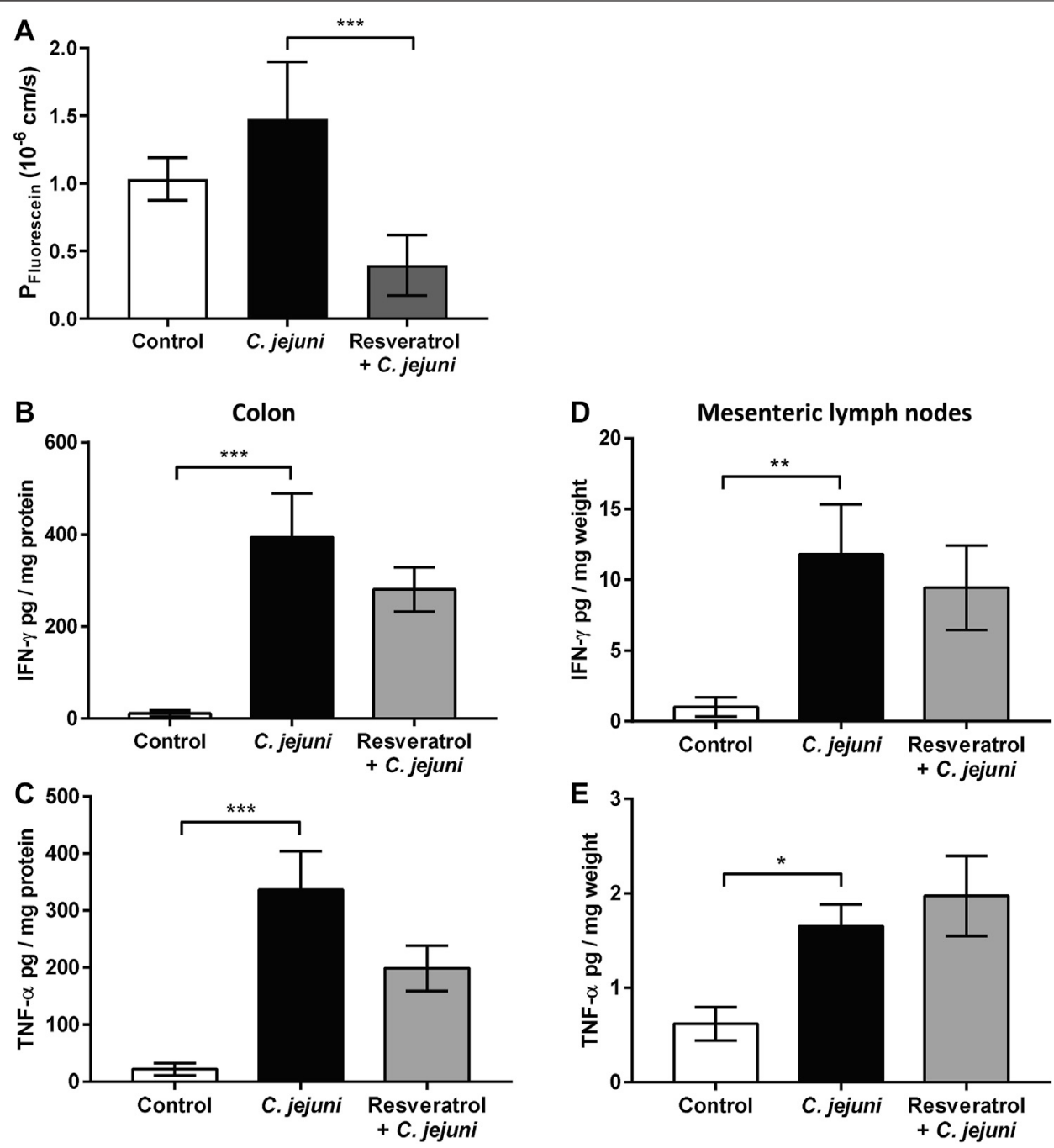

FIGURE 7 | In vivo mouse infection model and oral treatment with resveratrol. Barrier function and simulated antigen influx by fluorescein in the Campylobacter jejuni-infected mouse colon. Secondary abiotic IL-10 ${ }^{-/-}$mice were infected with C. jejuni, treated with resveratrol via the drinking water and sacrificed at day 6 postinfection (A) Colon specimens of $C$. jejuni-infected and resveratrol treated mice were mounted into Ussing chambers and flux measurements of fluorescein were performed in mucosal to serosal direction (B and $\mathbf{C})$ release of pro-inflammatory cytokines of mouse organs from colon and (D and E) mesenteric lymph nodes. Organ specimens were cultured in cell culture medium and supernatant was analyzed by Cytometric Bead Array for (B and D) IFN- $\gamma$ and (C and E) TNF- $\alpha$ concentrations $\left(n=4-17,{ }^{*} p<0.05,{ }^{* *} p<0.01,{ }^{\star \star *} p<0.001\right.$, Mann Whitney U-test).

epithelial barrier function following C. jejuni infection and is therefore a promising nutraceutical. This suitability was predicted in the bioinformatics RNA-Seq data from campylobacteriosis patients by Ingenuity Pathway Analysis software. The Ingenuity Pathway Analysis prediction tool for activated or inhibited pathways and potentially active compounds (upstream analysis) in different diseases compares differential expressed genes in our Campylobacter dataset with a database of numerous available expression datasets from the literature. The information from the literature enables predictions not only for the pathogenesis and the significant or associated signaling pathways, but also on possible compounds that were already found to be effective in other diseases with similar patterns of RNA expression changes to that in campylobacteriosis. Although ancient knowledge from traditional healing arts points to the usefulness of plants containing resveratrol, questions about pharmacokinetics, bioavailability and enteral stability, as well as the adverse effects of resveratrol preparations, need to be addressed by clinical trials.

\section{Apoptosis Induction and Tight Junction Protein Changes in Campylobacter Infection and Resveratrol Treatment}

The anti-apoptotic effect of resveratrol is of high importance. The C. jejuni infection not only affects the TJ disruption- 
dependent leak pathway, but also the unrestricted pathway by barrier-relevant cytotoxicity. In our cell monolayer infection experiments, the ratio of apoptotic events was increased 3-fold and could be reduced to control values in the resveratrol treated group. An increase in apoptosis to this extent in an epithelial layer is barrier-relevant (Bojarski et al., 2004; Butkevych et al., 2020) and can explain, at least in part, the functional barrier defects as quantified by our measurements of TER and fluorescein permeability. Anti-apoptotic effects of resveratrol have been reported in $\mathrm{H}_{2} \mathrm{O}_{2}$-stimulated Caco-2 cells (Wang et al., 2016), and were confirmed in the present study. Campylobacter jejuni-infected $\mathrm{IL}-10^{-/-}$mice treated with resveratrol showed lower apoptotic cell numbers in the colon when compared with placebo-treated $C$. jejuni-infected mice (Heimesaat et al., 2020). Nevertheless, several studies and clinical trials showed that resveratrol also induced apoptosis in cancer cells (Berman et al., 2017; Singh et al., 2017; El-Kott et al., 2019). Besides the induction of apoptosis induction, the other main component of the functional barrier impairment can be explained by changes in TJ protein expression and subcellular redistribution of TJ proteins (Chen et al., 2006; Bücker et al., 2018). After infection with C. jejuni, HT-29/B6 cell monolayers showed a redistribution of barrier-maintaining occludin and claudin-5 off the TJ domain into intracellular compartments of the epithelial cells. A similar pattern of redistribution of occludin and claudin- 5 has been reported in other in vitro infection experiments with C. jejuni (Chen et al., 2006; Rees et al., 2008; Lobo de Sá et al., 2019) and in colon tissues from campylobacteriosis patients (Bücker et al., 2018; Harrer et al., 2019). This redistribution will contribute to epithelial barrier dysfunction via the paracellular leak pathway, and provides the structural basis of the mechanism underlying leak flux diarrhea (Bücker et al., 2018).

A discrepancy was seen in our Western blot studies of the downregulation of channel-forming claudin-2 and the upregulation of barrier-forming claudin- 1 . The claudin-1 paradox has previously been described in C. fetus and C. jejuni infection, in which claudin-1 showed increased expression (while TER was decreased). The explanation for this paradox was that claudin- 1 does not functionally assemble into the epithelial $\mathrm{TJ}$ strands but remains in intracellular compartments (Bücker et al., 2017; Bücker et al., 2018; Lobo de Sá et al., 2019; Butkevych et al., 2020). This can be interpreted as the start of a counter-reaction or recovery reaction 1-2 days post infection after the primary $\mathrm{TJ}$ dysregulation in the first phase of infection. By contrast, claudin-2 downregulation is assumed to be functionally masked by the other structural alterations.

\section{Campylobacter jejuni Infection in Stimulated Cell Cultures in vitro and Campylobacteriosis in Mice}

Pro-inflammatory cytokines such as TNF- $\alpha$ (Amasheh et al., 2010; Luettig et al., 2016) trigger dysregulation of TJ proteins as well as inducing apoptosis, and the mucosal cytokine storm is prominent in the pathogenesis of campylobacteriosis (Al-Salloom et al., 2003; Edwards et al., 2010; Bücker et al., 2018). We therefore investigated the role of cytokines in causing barrier dysfunction during C. jejuni infection in leaky gut models.

Leaky gut models to study Campylobacter infection have previously employed monolayers of human colon cell lines cocultured with M1-macrophages derived from human PBMCs (Bücker et al., 2018), or epithelial monolayers co-cultured with the stimulated macrophage-like cell line THP-1 (Lobo de Sá et al., 2019; Butkevych et al., 2020). Cell culture filter supports or transwell systems allow application of bacteria, cytokines, compounds or inhibitors from the apical or basolateral surfaces. For example, when investigating the immunemodulating effect of curcumin on the C. jejuni infection, basolateral located THP-1 cells were infected directly, without infecting the epithelial cell monolayers, simulating cytokineinduced impairment of the barrier function (Lobo de Sá et al., 2019). In this model, the addition of curcumin abolished barrier dysfunction in an NFkB-dependent manner (Lobo de Sá et al., 2019). The THP-1 model could not be applied in the present study as in this blood cancer cell line, resveratrol causes cell cycle arrest and induces apoptosis (Feng et al., 2019). For this reason, an alternative model with the addition of cytokines was used. Since Rees and colleagues showed that $100 \mathrm{U} / \mathrm{mL}$ IFN- $\gamma$ accelerates barrier impairment synergistically with $C$. jejuni in infected Caco-2 cells (Rees et al., 2008), and our Ingenuity Pathway analysis indicated the importance of the IFN- $\gamma$ pathway, we stimulated HT-29/B6 monolayers with IFN- $\gamma$ $(50 \mathrm{ng} / \mathrm{ml})$. We found that resveratrol antagonized the IFN$\gamma$-pathway resulting in an improvement of barrier function. In further experiments, barrier defects after maximal stimulation with a cytokine cocktail of TNF- $\alpha$, IL- $1 \beta$ and IFN- $\gamma$ could not be antagonized by resveratrol. This suggests that the pharmacological efficacy of resveratrol is primarily barrierimproving rather than immunomodulatory.

In addition to co-culture infection and direct cytokine exposure, the use of animal models is the standard method to gain information about the entire immune system after intestinal barrier breakdown during enteropathogenic infection (Gölz et al., 2015; von Klitzing et al., 2017; Heimesaat et al., 2019). In our present mouse model of campylobacteriosis, infected secondary abiotic IL-10 $0^{-/-}$mice suffered from acute enterocolitis within one week after infection (Haag et al., 2012), as indicated by bloody diarrhea and wasting. The underlying massive immune overreaction is based on the subepithelial cytokine storm induced by the LOS of the invasive campylobacters, suggesting that the pathogenicity of C. jejuni is predominantly based on LOS translocation into the subepithelium, whereby the influx of further LOS from the lumen in the leaky gut situation may amplifies mucosal inflammation. Given that the LOS of $C$. jejuni targets the toll-like receptor 4 (TLR4), the major role of LOS in the induction and progress of campylobacteriosis was supported by the fact that $C$. jejuni infection induced significantly milder enterocolitis symptoms in TLR4-deficient mice. This was confirmed independently by different research groups in their individual murine models of camyplobacteriosis (Otto et al., 2012; Haag et al., 2012; Stahl et al., 2014; Stahl \& Vallance, 2015; reviewed by Mousavi et al., 2020). Most importantly, a 
general pharmacological mechanism of action against the inflammation induced by $C$. jejuni includes the blockage or antagonism of TLR4 signaling pathways, which should counter-regulate the pathological consequences of the leaky gut. This kind of immune induction via a TLR4-dependent pathway might be inhibited by resveratrol in our mouse experiments. In support of this notion, C. jejuni-induced inflammatory responses were significantly decreased by the polyphenol curcumin (Lobo de Sá et al., 2019), which is a potent TLR4 antagonist and inhibits LOS-mediated immune responses (Zhao et al., 2011).

However, the direct impact of resveratrol on cytokine pathways appears to be effective only in the case of moderate barrier dysfunction, such as that induced by IFN- $\gamma$ treatment. Similar results were obtained in lymphocytes, in which cytokine production increased rather than decreased after resveratrol treatment (Gao et al., 2001). By contrast, production of IL-6 was downregulated in macrophages after resveratrol treatment (Zhong et al., 1999) whereas in mice with Dextran Sodium Sulfate (DSS)-induced colitis resveratrol decreased IL-6 release but increased TNF- $\alpha$ release (Mayangsari and Suzuki, 2018). Nevertheless, resveratrol restored intestinal barrier function in mice with DSS-induced colitis (Mayangsari and Suzuki, 2018). One interpretation of our results is that resveratrol does not function as an immunosuppressive agent, but rather as an immune-modulating compound acting on weakly affected cytokine pathways such as that dependent on IL-6. Therefore, the C. jejuni LOS-induced immune activation of TLR4, as occurs in our mouse model, might be modulated or in part inhibited by resveratrol, thereby contributing to the restoration of barrier dysfunction and avoidance of the leaky gut.

\section{Strengthening the Epithelial Barrier for Prevention of the Leaky gut}

Resveratrol has not only immune-modulatory functions, but also can reduce oxidative stress and influences several pathways in intestinal homeostasis (Singh et al., 2010; Yao et al., 2010; Yao et al., 2015; Cao et al., 2019). In our epithelial cell monocultures infected with C. jejuni, resveratrol exerted direct barrierimproving effects by preventing enhanced epithelial apoptosis and restoring TJ dysregulated claudins into the TJ domain. Furthermore, we speculate that epithelial defense mechanisms may be enhanced by resveratrol. In general, the mucosal defense against microorganisms can be modulated by increased expression of mucins, tight junctional proteins, secretion of chemokines and cytokines, or release of direct antimicrobials like $\beta$-defensins and cathelicidin. We know from other studies that resveratrol affects intracellular signaling pathways, for example, blockage of NFkB activation (Singh et al., 2010), activator protein-1 (Kundu and Surh, 2004), IкBa (Tsai et al., 1999) or TLR4 and STAT3 (Zhang et al., 2019). Notably, a recent study showed the synergistic effect of resveratrol and vitamin $\mathrm{D}$ on the heterodimerization of VDR-RXR nuclear factors, which reflected an increase in vitamin $\mathrm{D}$-dependent gene expression involving transactivation by resveratrol (Dampf Stone et al.,
2015). We have previously shown this vitamin D-dependent pathway to be affected in campylobacteriosis and provided the first evidence that supplementation with active vitamin D antagonized the effects of $C$. jejuni in vitro and to some extent in vivo (Bücker et al., 2018; Mousavi et al., 2019). It would therefore seem reasonable that novel therapeutic approaches should involve a combination of compounds, in order to use their synergistic effects to increase their overall efficacy. Agents that inhibit transepithelial migration of $C$. jejuni may also be valuable. And it is interesting that resveratrol reduced parasite translocation in a mouse model of intestinal Toxoplasma gondii infection (Bereswill et al., 2010). Thus, a barrier-improving agent such as resveratrol could be combined with an immune modulator such as curcumin or vitamin D.

In conclusion, the effects of $C$. jejuni infection can be attenuated by agents with barrier-protective, anti-inflammatory or anti-apoptotic actions, alone or in combination. Promising compounds to treat or prevent Campylobacter infections are nutraceuticals such as resveratrol or curcumin, which should be considered as therapeutic options in multimodal approaches to treat acute enteritis or post-infective IBS. Resveratrol is particularly effective in restoring the epithelial leak pathway and may prevent the pathogenesis of a leaky gut.

\section{DATA AVAILABILITY STATEMENT}

The raw data supporting the conclusions of this article will be made available by the authors, without undue reservation.

\section{ETHICS STATEMENT}

The studies involving human participants were reviewed and approved by the Ethics Committee of the Charité ${ }^{\odot}$ (Berlin, Germany). The patients/participants provided their written informed consent to participate in this study.

The animal study was reviewed and approved by the ethics committee for animal welfare in Berlin, Landesamt für Gesundheit und Soziales (LAGeSo Berlin), Germany.

\section{AUTHOR CONTRIBUTIONS}

Conceptualization, FL and RB; Data curation, $\mathrm{MMH}, \mathrm{FL}, \mathrm{PN}$; Formal analysis, JDS, FL, SB; Funding acquisition, RB, JDS, SB, $\mathrm{MMH}$; Investigation, FL, PN, MMH; Methodology, RB, SB, JDS; Project administration, RB; Supervision, RB, JDS; Writing-original draft, RB, FL; Writing_review and editing, JDS, MMH, SB.

\section{FUNDING}

We acknowledge support from the German Research Foundation and the Open Access Publication Fund of the Charité-Universitätsmedizin Berlin. This work was supported by the German Federal Ministry of Education and Research (BMBF) in 
frame of the zoonoses research consortium PAC-Campylobacter to $\mathrm{RB}$ and JDS (project IP8/01KI2007D) and to $\mathrm{MMH}$ and SB (IP7/ $01 \mathrm{KI} 1725 \mathrm{D})$. This work was supported by the German Research Foundation (DFG Schu559/11-3) to JDS. Furthermore, MMH and $\mathrm{SB}$ received support from the German Federal Ministries of Economy and Energy (ZIM; ZF4117904 AJ8).

\section{REFERENCES}

Al-Salloom, F., Mahmeed, A. A., Ismaeel, A., Botta, G. A., and Bakhiet, M. (2003). Campylobacter-stimulated INT407 cells produce dissociated cytokine profiles. J. Infect. 47 (3), 217-224. doi:10.1016/s0163-4453(03)00076-8

Amasheh, M., Fromm, A., Krug, S. M., Amasheh, S., Andres, S., Zeitz, M., et al. (2010). TNFa-induced and berberine-antagonized tight junction barrier impairment via tyrosine kinase, Akt and NFkB signaling. J. Cell Sci. 123 (23), 4145-4155. doi:10.1242/jcs.070896

Bereswill, S., Muñoz, M., Fischer, A., Plickert, R., Haag, L.-M., Otto, B., et al. (2010). Anti-inflammatory effects of resveratrol, curcumin and simvastatin in acute small intestinal inflammation. PLoS One 5 (12), e15099. doi:10.1371/journal. pone.0015099

Bergann, T., Plöger, S., Fromm, A., Zeissig, S., Borden, S. A., Fromm, M., et al. (2009). A colonic mineralocorticoid receptor cell model expressing epithelial $\mathrm{Na}+$ channels. Biochem. Biophys. Res. Comm. 381 (2), 280-285. doi:10.1016/j. bbrc.2009.03.006

Berman, A. Y., Motechin, R. A., Wiesenfeld, M. Y., and Holz, M. K. (2017). The therapeutic potential of resveratrol: a review of clinical trials. npj Precision Onc 1, 35. doi:10.1038/s41698-017-0038-6

Black, R. E., Levine, M. M., Clements, M. L., Hughes, T. P., and Blaser, M. J. (1988). Experimental Campylobacter jejuni infection in humans. J. Infect. Dis. 157 (3), 472-479. doi:10.1093/infdis/157.3.472

Bojarski, C., Weiske, J., Schöneberg, T., Schröder, W., Mankertz, J., Schulzke, J. D., et al. (2004). The specific fates of tight junction proteins in apoptotic epithelial cells. J. Cell Sci. 117 (10), 2097-2107. doi:10.1242/jcs.01071

Bücker, R., Krug, S. M., Fromm, A., Nielsen, H. L., Fromm, M., Nielsen, H., et al. (2017). Campylobacter fetusimpairs barrier function in HT-29/B6 cells through focal tight junction alterations and leaks. Ann. N.Y. Acad. Sci. 1405 (1), 189-201. doi:10.1111/nyas.13406

Bücker, R., Krug, S. M., Moos, V., Bojarski, C., Schweiger, M. R., Kerick, M., et al. (2018). Erratum: Campylobacter jejuni impairs sodium transport and epithelial barrier function via cytokine release in human colon. Mucosal Immunol. 11 (2), 575-577. doi:10.1038/mi.2017.78

Bücker, R., Schulz, E., Günzel, D., Bojarski, C., Lee, I.-F. M., John, L. J., et al. (2014). $\alpha$-Haemolysin ofEscherichia coliin IBD: a potentiator of inflammatory activity in the colon. Gut 63 (12), 1893-1901. doi:10.1136/gutjnl-2013-306099

Butkevych, E., Lobo de Sá, F. D., Nattramilarasu, P. K., and Bücker, R. (2020). Contribution of epithelial apoptosis and subepithelial immune responses in Campylobacter jejuni-induced barrier disruption. Front. Microbiol. 11, 344. doi:10.3389/fmicb.2020.00344

Cao, S., Shen, Z., Wang, C., Zhang, Q., Hong, Q., He, Y., et al. (2019). Resveratrol improves intestinal barrier function, alleviates mitochondrial dysfunction and induces mitophagy in diquat challenged piglets1. Food Funct. 10 (1), 344-354. doi:10.1039/c8fo02091d

Carrasco-Pozo, C., Morales, P., and Gotteland, M. (2013). Polyphenols protect the epithelial barrier function of Caco-2 cells exposed to indomethacin through the modulation of occludin and zonula occludens-1 expression. J. Agric. Food Chem. 61 (22), 5291-5297. doi:10.1021/jf400150p

Chen, M. L., Ge, Z., Fox, J. G., and Schauer, D. B. (2006). Disruption of tight junctions and induction of proinflammatory cytokine responses in colonic epithelial cells by Campylobacter jejuni. Iai 74 (12), 6581-6589. doi:10.1128/IAI.00958-06

Chudzińska, M., Rogowicz, D., Wołowiec, Ł., Banach, J., Sielski, S., Bujak, R., et al. (2020). Resveratrol and cardiovascular system-the unfulfilled hopes. Ir J. Med. Sci. doi:10.1007/s11845-020-02441-x

Dampf Stone, A., Batie, S. F., Sabir, M. S., Jacobs, E. T., Lee, J. H., Whitfield, G. K., et al. (2015). Resveratrol potentiates vitamin $\mathrm{D}$ and nuclear receptor signaling. J. Cell. Biochem. 116 (6), 1130-1143. doi:10.1002/jcb.25070

\section{ACKNOWLEDGMENTS}

We acknowledge the excellent technical support of Anja Fromm and In-Fah Maria Lee. We wish to thank Professor Geoffrey Sandle (University of Leeds, United Kingdom) for proof reading and editing the manuscript.

Edwards, L. A., Nistala, K., Mills, D. C., Stephenson, H. N., Zilbauer, M., Wren, B. W., et al. (2010). Delineation of the innate and adaptive T-cell immune outcome in the human host in response to Campylobacter jejuni infection. PLoS One 5 (11), e15398. doi:10.1371/journal.pone.0015398

El-Kott, A. F., Shati, A. A., Ali Al-Kahtani, M., and Alharbi, S. A. (2019). The apoptotic effect of resveratrol in ovarian cancer cells is associated with downregulation of galectin-3 and stimulating miR-424-3p transcription. J. Food Biochem. 43 (12), e13072. doi:10.1111/jfbc.13072

Feng, L., Yasmeen, R., Schoene, N. W., Lei, K. Y., and Wang, T. T. Y. (2019). Resveratrol differentially modulates immune responses in human THP-1 monocytes and macrophages. Nutr. Res. 72, 57-69. doi:10.1016/j.nutres. 2019.10.003

Gao, X., Xu, Y. X., Janakiraman, N., Chapman, R. A., and Gautam*, S. C. (2001). Immunomodulatory activity of resveratrol: suppression of lymphocyte proliferation, development of cell-mediated cytotoxicity, and cytokine production11Abbreviations: CTLs, cytotoxic T lymphocytes; LAK cells, lymphokine activated killer cells; IL-2, interleukin-2; IFN- $\gamma$, interferongamma; TNF- $\alpha$, tumor necrosis factor- $\alpha$ NF- $\kappa B$, nuclear factor kappa B; Con A, concanavalin A; HBSS, Hanks' balanced salt solution; DTT, dithiothreitol; PMSF, phenylmethylsulfonyl fluoride; RT-PCR, reverse transcription-polymerase chain reaction; LPS, lipopolysaccharide; and EMSA, electrophoretic mobility shift assay. Biochem. Pharmacol. 62, 1299-1308. doi:10.1016/s0006-2952(01)00775-4

Gölz, G., Karadas, G., Alutis, M. E., Fischer, A., Kühl, A. A., Breithaupt, A., et al. (2015). Arcobacter butzleri induce colonic, extra-intestinal and systemic inflammatory responses in Gnotobiotic IL-10 deficient mice in a strain-dependent manner. PLoS One 10 (9), e0139402. doi:10.1371/journal.pone.0139402

Haag, L.-M., Fischer, A., Otto, B., Plickert, R., Kühl, A. A., Göbel, U. B., et al. (2012). Campylobacter jejuni induces acute enterocolitis in Gnotobiotic IL-10-/- mice via toll-like-receptor-2 and -4 signaling. PLoS One 7 (7), e40761. doi:10.1371/ journal.pone.0040761

Harrer, A., Bücker, R., Boehm, M., Zarzecka, U., Tegtmeyer, N., Sticht, H., et al. (2019). Campylobacter jejuni enters gut epithelial cells and impairs intestinal barrier function through cleavage of occludin by serine protease HtrA. Gut Pathog. 11, 4. doi:10.1186/s13099-019-0283-Z

Heimesaat, M. M., Escher, U., Grunau, A., Kühl, A. A., and Bereswill, S. (2019). Multidrug-resistant Pseudomonas aeruginosa accelerate intestinal, extraintestinal, and systemic inflammatory responses in human microbiotaassociated mice with subacute ileitis. Front. Immunol. 10, 49. doi:10.3389/ fimmu.2019.00049

Heimesaat, M. M., Mousavi, S., Escher, U., Lobo de Sá, F. D., Peh, E., Schulzke, J.D., et al. (2020). Resveratrol alleviates acute Campylobacter jejuni induced enterocolitis in a preclinical murine intervention study. Microorganisms 8 (12), 1858. doi:10.3390/microorganisms 8121858

Hollander, D. (1999). Intestinal permeability, leaky gut, and intestinal disorders. Curr. Gastroenterol. Rep. 1 (5), 410-416. doi:10.1007/s11894 999-0023-5

Klančnik, A., Šikić Pogačar, M., Trošt, K., Tušek Žnidarič, M., Mozetič Vodopivec, B., and Smole Možina, S. (2017). Anti-Campylobacter activity of resveratrol and an extract from waste Pinot noir grape skins and seeds, and resistance of Camp. jejuni planktonic and biofilm cells, mediated via the CmeABC efflux pump. J. Appl. Microbiol. 122 (1), 65-77. doi:10.1111/jam.13315

Koushki, M., Amiri-Dashatan, N., Ahmadi, N., Abbaszadeh, H. A., and RezaeiTavirani, M. (2018a). Resveratrol: a miraculous natural compound for diseases treatment. Food Sci. Nutr. 6 (8), 2473-2490. doi:10.1002/fsn3.855

Koushki, M., Dashatan, N. A., and Meshkani, R. (2018b). Effect of resveratrol supplementation on inflammatory markers: a systematic review and metaanalysis of randomized controlled trials. Clin. Ther. 40 (7), 1180-1192. doi:10. 1016/j.clinthera.2018.05.015 
Kundu, J. K., and Surh, Y.-J. (2004). Molecular basis of chemoprevention by resveratrol: NF- $\mathrm{\kappa B}$ and AP-1 as potential targets. Mutat. Research/Fundamental Mol. Mech. Mutagenesis 555 (1-2), 65-80. doi:10.1016/j.mrfmmm.2004.05.019

Ling, K.-H., Wan, M. L. Y., El-Nezami, H., and Wang, M. (2016). Protective capacity of resveratrol, a natural polyphenolic compound, against deoxynivalenol-induced intestinal barrier dysfunction and bacterial translocation. Chem. Res. Toxicol. 29 (5), 823-833. doi:10.1021/acs. chemrestox.6b00001

Liu, J., Zhang, X., Yan, T., Wang, F., Li, J., Jia, L., et al. (2020). Screening of an endophyte transforming polydatin to resveratrol from Reynoutria japonica houtt and the optimization of its transformation parameters. Molecules 25 (20), 4830. doi:10.3390/molecules 25204830

Lobo de Sá, F. D., Butkevych, E., Nattramilarasu, P. K., Fromm, A., Mousavi, S., Moos, V., et al. (2019). Curcumin mitigates immune-induced epithelial barrier dysfunction by Campylobacter jejuni. Int. J. Mol. Sci. 20 (19), 4830. doi:10.3390/ ijms 20194830

Lobo de Sá, F. D., Schulzke, J. D., and Bücker, R. (2021). Diarrheal mechanisms and the role of intestinal barrier dysfunction in Campylobacter infections. Curr. Top. Microbiol. Immunol. 431, 203-231. doi:10.1007/978-3-030-65481-8_8

Luettig, J., Rosenthal, R., Lee, I.-F. M., Krug, S. M., and Schulzke, J. D. (2016). The ginger component 6-shogaol prevents TNF- $\alpha$-induced barrier loss via inhibition of PI3K/Akt and NF-KB signaling. Mol. Nutr. Food Res. 60 (12), 2576-2586. doi:10.1002/mnfr.201600274

Mayangsari, Y., and Suzuki, T. (2018). Resveratrol ameliorates intestinal barrier defects and inflammation in colitic mice and intestinal cells. J. Agric. Food Chem. 66 (48), 12666-12674. doi:10.1021/acs.jafc.8b04138

Mousavi, S., Bereswill, S., and Heimesaat, M. (2020). Novel clinical Campylobacter jejuni infection models based on sensitization of mice to lipooligosaccharide, a major bacterial factor triggering innate immune responses in human campylobacteriosis. Microorganisms 8 (4), 482. doi:10.3390/ microorganisms 8040482

Mousavi, S., Lobo de Sá, F. D., Schulzke, J.-D., Bücker, R., Bereswill, S., and Heimesaat, M. M. (2019). Vitamin D in acute campylobacteriosis-results from an intervention study applying a clinical Campylobacter jejuni induced enterocolitis model. Front. Immunol. 10, 2094. doi:10.3389/fimmu.2019.02094

Nielsen, H. L., Engberg, J., Ejlertsen, T., Bücker, R., and Nielsen, H. (2012). Short-term and medium-term clinical outcomes of Campylobacter concisus infection. Clin. Microbiol. Infect. 18 (11), E459-E465. doi:10.1111/j.1469-0691.2012.03990.x

Otto, B., Haag, L.-M., Fischer, A., Plickert, R., Kühl, A. A., Göbel, U. B., et al. (2012). Campylobacter jejuniinduces extra-intestinal immune responses via toll-likereceptor-4 signaling in conventional IL-10 deficient mice with chronic colitis. Eur. J. Microbiol. Immunol. (Bp) 2 (3), 210-219. doi:10.1556/EuJMI.2.2012.3.7

Rees, L. E. N., Cogan, T. A., Dodson, A. L., Birchall, M. A., Bailey, M., and Humphrey, T. J. (2008). Campylobacter and IFN $\gamma$ interact to cause a rapid loss of epithelial barrier integrity. Inflamm. Bowel Dis. 14 (3), 303-309. doi:10.1002/ibd.20325

Rosenthal, R., Luettig, J., Hering, N. A., Krug, S. M., Albrecht, U., Fromm, M., et al. (2017). Myrrh exerts barrier-stabilising and -protective effects in HT-29/B6 and Caco-2 intestinal epithelial cells. Int. J. Colorectal Dis. 32 (5), 623-634. doi:10. 1007/s00384-016-2736-x

Salehi, B., Mishra, A., Nigam, M., Sener, B., Kilic, M., Sharifi-Rad, M., et al. (2018). Resveratrol: a double-edged sword in health benefits. Biomedicines 6 (3), 91. doi:10.3390/biomedicines6030091

Schulzke, J.-D., Bojarski, C., Zeissig, S., Heller, F., Gitter, A. H., and Fromm, M. (2006). Disrupted barrier function through epithelial cell apoptosis. Ann. New York Acad. Sci. 1072, 288-299. doi:10.1196/annals.1326.027

Shane, S. M. (1992). The significance ofcampylobacter jejuniinfection in poultry: a review. Avian Pathol. 21 (2), 189-213. doi:10.1080/03079459208418836

Singh, S. K., Banerjee, S., Acosta, E. P., Lillard, J. W., and Singh, R. (2017). Resveratrol induces cell cycle arrest and apoptosis with docetaxel in prostate cancer cells via a p53/p21WAF1/CIP1 and p27KIP1 pathway. Oncotarget 8 (10), 17216-17228. doi:10.18632/oncotarget.15303
Singh, U. P., Singh, N. P., Singh, B., Hofseth, L. J., Price, R. L., Nagarkatti, M., et al. (2010). Resveratrol (Trans-3,5,4'-trihydroxystilbene) induces silent mating type information regulation-1 and down-regulates nuclear transcription factor-kb activation to abrogate dextran sulfate sodium-induced colitis. J. Pharmacol. Exp. Ther. 332 (3), 829-839. doi:10.1124/jpet.109.160838

Stahl, M., Ries, J., Vermeulen, J., Yang, H., Sham, H. P., Crowley, S. M., et al. (2014). A novel mouse model of Campylobacter jejuni gastroenteritis reveals key proinflammatory and tissue protective roles for Toll-like receptor signaling during infection. Plos Pathog. 10 (7), e1004264. doi:10.1371/journal.ppat.1004264

Stahl, M., and Vallance, B. A. (2015). Insights intoCampylobacter jejunicolonization of the mammalian intestinal tract using a novel mouse model of infection. Gut Microbes 6 (2), 143-148. doi:10.1080/19490976.2015.1016691

Tsai, S. H., Lin-Shiau, S. Y., and Lin, J. K. (1999). Suppression of nitric oxide synthase and the down-regulation of the activation of NFKB in macrophages by resveratrol. Br. J. Pharmacol. 126 (3), 673-680. doi:10.1038/sj.bjp.0702357

Vandamme, P., Falsen, E., Rossau, R., Hoste, B., Segers, P., Tytgat, R., et al. (1991). Revision of Campylobacter, Helicobacter, and Wolinella taxonomy: emendation of generic descriptions and proposal of Arcobacter gen. nov. Int. J. Syst. Bacteriol. 41 (1), 88-103. doi:10.1099/00207713-41-1-88

von Klitzing, E., Ekmekciu, I., Kühl, A. A., Bereswill, S., and Heimesaat, M. M. (2017). Intestinal, extra-intestinal and systemic sequelae of Toxoplasma gondii induced acute ileitis in mice harboring a human gut microbiota. PLoS One 12 (4), e0176144. doi:10.1371/journal.pone.0176144

Wang, N., Han, Q., Wang, G., Ma, W. P., Wang, J., Wu, W. X., et al. (2016). Resveratrol protects oxidative stress-induced intestinal epithelial barrier dysfunction by upregulating heme oxygenase-1 expression. Dig. Dis. Sci. 61 (9), 2522-2534. doi:10.1007/s10620-016-4184-4

Wassenaar, T. M., and Blaser, M. J. (1999). Pathophysiology of Campylobacter jejuni infections of humans. Microbes Infect. 1 (12), 1023-1033. doi:10.1016/ s1286-4579(99)80520-6

Weiskirchen, S., and Weiskirchen, R. (2016). Resveratrol: how much wine do you have to drink to stay healthy?. Adv. Nutr. 7 (4), 706-718. doi:10.3945/an.115.011627

Yao, J., Wang, J. Y., Liu, L., Li, Y. X., Xun, A. Y., Zeng, W. S., et al. (2010). Antioxidant effects of resveratrol on mice with DSS-induced ulcerative colitis. Arch. Med. Res. 41 (4), 288-294. doi:10.1016/j.arcmed.2010.05.002

Yao, J., Wei, C., Wang, J. Y., Zhang, R., Li, Y. X., and Wang, L. S. (2015). Effect of resveratrol on Treg/Th17 signaling and ulcerative colitis treatment in mice. Wjg 21 (21), 6572-6581. doi:10.3748/wjg.v21.i21.6572

Zhang, M., Xue, Y., Chen, H., Meng, L., Chen, B., Gong, H., et al. (2019). Resveratrol inhibits MMP3 and MMP9 expression and secretion by suppressing TLR4/NF-KB/STAT3 activation in ox-LDL-treated HUVECs. Oxid Med. Cell Longev, 1. doi:10.1155/2019/9013169

Zhao, L., Lee, J. Y., and Hwang, D. H. (2011). Inhibition of pattern recognition receptor-mediated inflammation by bioactive phytochemicals. Nutr. Rev. 69 (6), 310-320. doi:10.1111/j.1753-4887.2011.00394.x

Zhong, M., Cheng, G. F., Wang, W. J., Guo, Y., Zhu, X. Y., and Zhang, J. T. (1999). Inhibitory effect of resveratrol on interleukin 6 release by stimulated peritoneal macrophages of mice. Phytomedicine 6 (2), 79-84. doi:10.1016/S0944-7113(99) 80039-7

Conflict of Interest: The authors declare that the research was conducted in the absence of any commercial or financial relationships that could be construed as a potential conflict of interest.

Copyright $\odot 2021$ Lobo de Sá, Heimesaat, Bereswill, Nattramilarasu, Schulzke and Bücker. This is an open-access article distributed under the terms of the Creative Commons Attribution License (CC BY). The use, distribution or reproduction in other forums is permitted, provided the original author(s) and the copyright owner(s) are credited and that the original publication in this journal is cited, in accordance with accepted academic practice. No use, distribution or reproduction is permitted which does not comply with these terms. 